ESAIM: COCV

Vol. 13, No 2, 2007, pp. 359-377

DOI: $10.1051 /$ cocv:2007016
ESAIM: Control, Optimisation and Calculus of Variations

www.edpsciences.org/cocv

\title{
THE FORMATION OF A TREE LEAF*
}

\author{
QingLAN XiA ${ }^{1}$
}

\begin{abstract}
In this article, we build a mathematical model to understand the formation of a tree leaf. Our model is based on the idea that a leaf tends to maximize internal efficiency by developing an efficient transport system for transporting water and nutrients. The meaning of "the efficient transport system" may vary as the type of the tree leave varies. In this article, we will demonstrate that tree leaves have different shapes and venation patterns mainly because they have adopted different efficient transport systems. The efficient transport system of a tree leaf built here is a modified version of the optimal transport path, which was introduced by the author in [Comm. Cont. Math. 5 (2003) 251-279; Calc. Var. Partial Differ. Equ. 20 (2004) 283-299; Boundary regularity of optimal transport paths, Preprint] to study the phenomenon of ramifying structures in mass transportation. In the present paper, the cost functional on transport systems is controlled by two meaningful parameters. The first parameter describes the economy of scale which comes with transporting large quantities together, while the second parameter discourages the direction of outgoing veins at each node from differing much from the direction of the incoming vein. Under the same initial condition, efficient transport systems modeled by different parameters will provide tree leaves with different shapes and different venation patterns. Based on this model, we also provide some computer visualization of tree leaves, which resemble many known leaves including the maple and mulberry leaf. It demonstrates that optimal transportation plays a key role in the formation of tree leaves.
\end{abstract}

Mathematics Subject Classification. 92B05, 49Q20,90B18.

Received November 28, 2005.

\section{INTRODUCTION}

Plant leaves have diverse and elaborate shapes and venation patterns. The beauty of them has attracted curiosity of many people involving biologists, physicists, mathematician, artists, computer scientists, etc., for a long time. Since the patterns of different species exhibit a large variety of structures, leaf venation has been investigated for its possible use in the systematic determination of species. Also, in palaeobotany, macrofossils showing leaf venation patterns are very helpful in identifying fossil taxa [9]. Current knowledge about leaf venation patterns mainly focus on their classification $[4,7,10]$, function [11] and their formation.

The aim of this article is to develop a geometric variational model for leaf shapes and leaf venation patterns. To understand the formation of a tree leaf, it is necessary to understand the main tasks of the tree leaf. A tree leaf will transport resources like water and solutes from its root to its tissues via xylem, absorb solar energy at

Keywords and phrases. Formation of a tree leaf, optimal transport system, leaf shape, leaf venation pattern.

* This work was supported by an NSF grant DMS-0306686. The author wants to express his thanks to Professors Luis Caffarelli and Robert Hardt for helpful discussions.

1 University of California at Davis, Mathematics, Davis, CA, 95616, USA; qlxia@math.ucdavis.edu 
their cells through photosynthesis, and then transport the chemical products (carbohydrates) synthesized in the leaf back to its root by phloem. Thus, a leaf tends to increase the surface area as large as possible to maximize metabolic capacity, because metabolism produces the energy and materials required to sustain and reproduce life. On the other hand, more importantly, the leaf tends to maximize internal efficiency by developing an efficient transporting system. One of the main tasks here is to describe this efficient biological transport system. In this article, a leaf is viewed as a union of square cells centered on a grid, and a transport system for the leaf is modeled by a weighted directed graph from the centers of the cells to the root. The cost function on the transport systems is modeled by a function of two reasonable parameters. One of them governs the phenomenon that transporting two items together might be cheaper than the total cost of transporting them separately. The other parameter says that transporting items along with the existing direction might be cheaper than rotating the angle and transporting them in different directions. In general, the cost function usually depends on the species of the tree leaf.

With a given cost function on transport systems, the formation of a leaf is mainly governed by a selection principle. As we know, the growing of a tree leaf is a dynamic process of generating new cells. It originates from a bud with a given initial growing direction. At every stage, the leaf will develop an optimal transport system to transport water between the root and the existing cells with respect to the given transport cost function. Also, as the environment changes, the leaf may generate some new cells nearby its boundary. The selection of those new cells is not random. Under the same environmental conditions, each potential new cell outside the existing leaf produces about the same amount of revenue such as the absorbed solar energy. However, the expense corresponding to each potential new cell varies with respect to the position of the cell. Here, the expense is mainly the transport cost of water and nutrients between the cell and the root. A selection principle says that a new cell is generated only if the expense is less than the revenue it produces. This simple rule determines the selection of new cells during the generation process. When the environmental conditions change, the corresponding revenue that a cell can produce also changes. When the corresponding revenue of each cell increased to a certain degree, it becomes benefit to produce some new cells, and thus the leaf will grow. Due to limited resources, the revenue that a cell can possibly produce is bounded above. This fact forces the leaf to stop growing after some time. As a result, the final shape and venation pattern of a leaf are mainly determined by the cost function defined on the collection of all possible transport systems, as well as the actual environment.

The article is organized as follows. We first discuss transport systems for an arbitrary subset of a given grid in Section 2. A transport system for such a subset is defined by a weighted directed graph satisfying a balancing equation at its vertices. Then we provide a reasonable two parameter family of cost functionals on the collection of all possible transport systems. We also provide a simple local algorithm on how to get an optimal transport system from an existing transport system. After that, we begin to study the growth of a tree leaf in Section 3. It originates from a bud with a given growing direction. At every stage, as environment changes, it generates new cells nearby its boundary. The selection of new cells is governed by the selection principle discussed above. Based on these ideas, some computer visualization of various leaves are provided in the end of the section. It turns out that this model provides the shapes and venation patterns of many well-known leaves. Changing the values of the parameters will give us different leaves. It demonstrates that internal efficiency plays a fundamental role in the formation of leaf shapes and associated venation patterns. In the end, we discuss a limiting process for our model by letting the size of the grid approach zero. The limiting leaf will correspond to a Radon measure with connected compact support, and its transport system becomes a vector measure whose divergence is the difference of the leaf (viewed as a measure) and the Dirac measure located at the root in the sense of distribution.

\section{OPTIMAL TRANSPORT SYSTEMS}

For simplicity, a leaf may be viewed as a finite union of distinct squares, centered on a given grid. The centers of these squares form a subset of the grid, but of course, not every subset of the grid comes from a leaf. The speciality of such subsets is closely related to the transport systems it adapted. For this reason, we first study the transport system on any subset of a given grid. 
Let $h>0$ be a fixed number. For any $m, n \in \mathbb{Z}$, the square

$$
C_{m, n}=[m h-h / 2, m h+h / 2) \times[n h-h / 2, n h+h / 2)
$$

is called a cell of size $h$, centered at the point $(m h, n h)$. Let

$$
\Gamma_{h}=\{(m h, n h): m, n \in \mathbb{Z}\}
$$

be the grid in $\mathbb{R}^{2}$ of size $h$ representing all possible locations of the centers of cells. The origin $O=(0,0) \in \Gamma_{h}$ is called the root. Let the unit vector $\vec{e}_{O}=(0,1)$ be the initial direction of $O$. That is, water initially follows out of the root $O$ in the direction $\vec{e}_{O}$.

Let $\Omega=\left\{x_{1}, x_{2}, \ldots, x_{k}\right\} \subset \Gamma_{h}$ be any finite subset representing a prospective leaf. To sustain and reproduce life, water needs to be transported from the root $O$ to cells centered at $x_{i}^{\prime} s$. The amount of water needed at each cell is proportional to its area. Without losing generality, we may assume it is $h^{2}$. So, each $x_{i} \in \Omega$ corresponds to a particle of mass $h^{2}$ located at $x_{i}$. We are looking for a way to transport water from the root $O$, flowing initially in the direction $\vec{e}_{O}$, to these particles.

To build a transport system for $\Omega$, we first recall some basic concepts from classical graph theory. As in [6], a directed graph $G$ consists of two finite sets $V(G)$ and $E(G)$. Each element of $V(G)$ is called a vertex and each element of $E(G)$, called an edge, is an ordered pair of vertices $(u, v)$. For each edge $e=(u, v) \in E(G)$, the unit directional vector of $e$ is denoted by $\vec{e}$, the vertex $u$ is denoted by $e^{-}$, and the vertex $v$ is denoted by $e^{+}$. A path in $G$ is a sequence of distinct vertices $\left\{v_{1}, v_{2}, \ldots, v_{k}\right\}$ such that $\left(v_{i}, v_{i+1}\right) \in E(G)$ for $i=1,2, \ldots, k-1$. A cycle in $G$ is a sequence of vertices $w_{1}, \ldots, w_{r}$ such that $w_{1}, \ldots, w_{r-1}$ is a path, $w_{1}=w_{r}$ and $\left(w_{r-1}, w_{r}\right) \in E(G)$.

Now we may introduce the following definition of transport systems of $\Omega$ :

Definition 2.1. A transport system of $\Omega$ is a weighted directed graph $G=\{V(G), E(G), w\}$ consists of a finite vertex set $V(G) \subset \Gamma_{h}$, a set $E(G)$ of directed edges and a weight function

$$
w: E(G) \rightarrow(0,+\infty)
$$

such that

(1) $\Omega \cup\{O\} \subset V(G)$.

(2) $G$ is connected and contains no cycles.

(3) The weight function $w: E(G) \rightarrow(0,+\infty)$ satisfies a balancing equation

$$
\sum_{\substack{e \in E(G) \\
e^{+}=v}} w(e)=\sum_{\substack{e \in E(G) \\
e^{-}=v}} w(e)+\left\{\begin{array}{cc}
h^{2}, & \text { if } v \in \Omega \\
0, & \text { otherwise }
\end{array}\right.
$$

at every vertex $v \in V(G) \backslash\{O\}$. That is, the total mass flows into $v$ equals to the total mass flows out of $v$.

Note that, for each vertex $v \in V(G) \backslash\{O\}$, since $G$ is connected and contains no cycles, there is a unique path

$$
P_{v}=\left\{v_{0}, v_{1}, \ldots, v_{l-1}, v_{l}\right\}
$$

from $O$ to $v$ such that $v_{0}=O$ and $v_{l}=v$ for some $l \in \mathbb{N}$. The vertex $v_{l-1} \in V(G)$ is called the parent of the vertex $v$, and denoted by $p(v)$. The directed edge $(p(v), v) \in E(G)$ is denoted by $e_{v}$. Thus, $e_{v}^{-}=p(v)$ and $e_{v}^{+}=v$. Also, one simply has

$$
E(G)=\left\{e_{v}: v \in V(G) \backslash\{O\}\right\}
$$


and the balancing equation (2.2) becomes

$$
w\left(e_{v}\right)=\sum_{\substack{e \in E(G) \\
e^{-}=v}} w(e)+\left\{\begin{array}{cc}
h^{2}, & \text { if } v \in \Omega \\
0, & \text { otherwise }
\end{array}\right.
$$

for each $v \in V(G) \backslash\{O\}$. Hence, the weight function $w$ is in fact determined whenever $V(G)$ and $E(G)$ are known.

For each vertex $v \in V(G)$, the set

$$
C_{v}=\{u \in V(G) \backslash\{O\}: p(u)=v\}
$$

is called the children set of $v$.

\subsection{Cost functional}

For a given $\Omega \subset \Gamma_{h}$, the collection of all transport systems of $\Omega$ may be a pretty large set, especially when the size of $\Omega$ is large. Among them, we are interested in finding a cost efficient one, which will be adapted by tree leaves as their venation. Thus, we need to find reasonable cost functionals on the collections of all possible transport systems. To find these cost functionals, we first make the following two crucial observations.

The first observation is that transporting two items together might be cheaper than the total cost of transporting them separately. For example in shipping two items from nearby cities to the same far away city, it may be less expensive to first bring them to a common location and put them on a single truck for most of the transport. In this case, a "Y shaped" path is preferable to a "V shaped" path. In general, a ramified structure might be more efficient than a straight line structure. Partially due to this reason, ramified structures are commonly adapted in many living and non-living systems. The author have modeled this phenomenon in [12-14] in terms of optimal transport paths. Over there, the author used the cost

$$
\mathbf{M}^{\alpha}(G):=\sum_{e \in E(G)}(w(e))^{\alpha} \text { length }(e)
$$

for any weighted directed graph $G=\{V(G), E(G), w\}$ between two atomic measures with a parameter $\alpha \in$ $(0,1)$, where length $(e)$ denotes the length of the edge $e$. Under this cost functional, a "Y shaped" path is preferable to a "V shaped" path, and ramified structures are preferable than straight line structures. Such an approach is meaningful and has many nice properties. For details, please see [12-14]. Some related work in this field maybe found in $[1-3,5,8]$.

The second observation is that when there exists a given transport direction, it is cheaper to transport items in the given direction than transport them in any other direction. That is, transportation tends to keep the existing flowing direction if any. For transporting the same amount of masses, it is cheaper to flow in the direction of the existing one than rotating the angle and move in other directions. The cost expenses will be an increasing function of the angle rotated. When angle rotates to more than $90^{\circ}$ degree, the cost becomes infinity. To take such reasonings into account, we introduce the following helping function. For any $\beta>0$, we consider the function

defined by

$$
H_{\beta}: \mathbb{S}^{1} \times \mathbb{S}^{1} \rightarrow(0,+\infty],
$$

$$
H_{\beta}(u, v)=\left\{\begin{array}{cc}
|u \cdot v|^{-\beta}, & \text { if } u \cdot v>0 \\
+\infty, & \text { otherwise }
\end{array}\right.
$$

for any pair of unit vectors $(u, v) \in \mathbb{S}^{1} \times \mathbb{S}^{1}$, where $\cdot$ is the usual dot product between vectors, and $\mathbb{S}$ is the unit circle in $\mathbb{R}^{2}$.

Thus, to model the growth of tree leaves, we modify the cost functional $\mathbf{M}^{\alpha}$ by adding some nonnegative scalar multiples to edges of transport systems. 
Let $G=\{V(G), E(G), w\}$ be any transport system of $\Omega$. For any given $\beta>0$, set

$$
m_{\beta}(O)=1 \text { and } \vec{e}_{O}=(0,1) .
$$

For each $v \in V(G)$, we set

$$
m_{\beta}(v)=m_{\beta}(p(v)) H_{\beta}\left(\vec{e}_{v}, \vec{e}_{p(v)}\right),
$$

where $p(v)$ is the parent vertex of $v$ defined after (2.3). Since $V(G)$ is finite and $G$ contains no cycles, such kind of recursive definition is well defined for all vertices $v \in V(G)$.

Based on the above observations, we introduce the following cost functional on the collection of transport systems.

Definition 2.2. Suppose $\alpha \in[0,1)$ and $\beta>0$ be two fixed real numbers. For any transport system $G=$ $\{V(G), E(G), w\}$ as above, the cost of $G$ is defined by

$$
\begin{aligned}
\mathbf{F}(G) & :=\sum_{e \in E(G)} m_{\beta}\left(e^{+}\right)(w(e))^{\alpha} \text { length }(e) \\
& =\sum_{v \in V(G) \backslash\{O\}} m_{\beta}(v)\left(w\left(e_{v}\right)\right)^{\alpha} \operatorname{length}\left(e_{v}\right) .
\end{aligned}
$$

From now on, we fix $\alpha$ and $\beta$, thus $\mathbf{F}$ is a fixed well defined functional on the collection of all possible transport systems. We are interested to see how a leaf grows from a single cell, after adapting the cost functional $\mathbf{F}$ on transport systems.

The following function measures the increment of the total cost if one add an extra mass of weight $x$ to the vertex $v \in V(G)$. Such a function is crucial in the dynamic process of generating new cells, and we will use it later.

Definition 2.3. Let $G$ be a transport system for $\Omega$. The potential function

$$
P_{G}:[0,+\infty) \times V(G) \rightarrow(0,+\infty]
$$

of $\Omega$ is defined by

$$
P_{G}(x, v)=\sum_{u \in P_{v} \backslash\{O\}} m_{\beta}(u)\left[\left(w\left(e_{u}\right)+x\right)^{\alpha}-\left(w\left(e_{u}\right)\right)^{\alpha}\right] \text { length }\left(e_{u}\right),
$$

for any $x \in[0,+\infty)$ and $v \in V(G) \backslash\{O\}$, where $P_{v}$ is the unique path in $G$ from $O$ to $v$ as in (2.3). Also, we set $P_{G}(x, O)=0$ for any $x \in[0,+\infty)$.

\subsection{Optimal transport systems}

Let $\Omega$ be any finite subset of $\Gamma_{h}$ as above, we are interested in looking for an optimal transport system for $\Omega$, which is a minimizer of $\mathbf{F}$ among all possible transport systems $G$ of $\Omega$. Since $\Omega$ is finite and the set of all possible transport systems of $\Omega$ is also finite, there exists an optimal transport path with least $\mathbf{F}$ cost. In general, it is still open to find an efficient algorithm which will give us the global (absolute) minimizer of $\mathbf{F}$, especially when the size of $\Omega$ is very large. Here, we provide a way to modify an existing transport system $G=\{V(G), E(G), w\}$. Since the growing of a leaf is a dynamic process, we want to keep the modified transport system not far away from that of the previous stage. A locally optimized transport system is suitable here.

The idea of the modification is straight forward: we fix the vertex set $V(G)$, and modify the edge set $E(G)$ by finding a "better parent" for each vertex of $G$ around the vertex as well as its current parent. To carrying on this idea, we provide the following algorithm: 
Step 1. For each vertex $v \in V(G)$, calculate the multiple $m_{\beta}(v)$ by $(2.6)$ as well as the cost of its "children"

$$
\operatorname{child}(v)=\sum_{c h \in C_{v}}\left(\operatorname{child}(c h)+w\left(e_{c h}\right)^{\alpha} m_{\beta}(c h) \text { length }\left(e_{c h}\right)\right),
$$

where $C_{v}$ is the children set of $v$ as defined in (2.5), and $e_{c h}$ is the edge $(v, c h) \in E(G)$. If $C_{v}=\emptyset$, then child $(v)=0$. Since $V(G)$ is finite and $G$ contains no cycles, such a recursive definition is well defined for all $v \in V(G)$.

Step 2. For any given $v \in V(G) \backslash\{O\}$, let

$$
\text { weight }=w\left(e_{v}\right)
$$

denote the weight of the edge $e_{v}$.

Substep 1. We remove the edge $e_{v}$ from $G$ by defining

$$
\tilde{w}(e)=\left\{\begin{array}{cc}
w(e)-w\left(e_{v}\right), & \text { if } e^{+} \in P_{v} \\
w(e), & \text { otherwise }
\end{array}\right.
$$

for each $e \in E(G) \backslash\left\{e_{v}\right\}$, where $P_{v}$ is defined in (2.3). That is, we simply change the values of $w$ for all edges $e$ with $e^{+} \in P_{v}$. Let $G_{0}$ be the modified graph.

Substep 2. Let

$$
Q_{v}=\{u:|v-u|<2 h \text { or }|v-p(v)|<2 h\} \backslash C_{v}
$$

be the admissible parent set of $v$, where $|\cdot|$ is the standard Euclidean distance in $\mathbb{R}^{2}$. We will choose a new parent in $Q_{v}$ for $v$ as follows.

For any $u \in Q_{v}$, we add an edge $(u, v)$ to the graph $G_{0}$ by set

$$
G_{u}=\left\{V(G),\left(E(G) \backslash\left\{e_{v}\right\}\right) \cup\{(u, v)\}, \bar{w}\right\},
$$

where

$$
\bar{w}(e)= \begin{cases}\tilde{w}(e)+w\left(e_{v}\right), & \text { if } e^{+} \in P_{u} \\ w\left(e_{v}\right), & e=(u, v) \\ \tilde{w}(e), & \text { otherwise }\end{cases}
$$

for each $e \in\left(E(G) \backslash\left\{e_{v}\right\}\right) \cup\{(u, v)\}$. Note that $G_{u}$ is again a transport system of $\Omega$. Let

$$
n_{u}=\left\{\begin{array}{cc}
\frac{v-u}{\mid v-u}, & \text { if } v \neq u \\
\vec{e}_{u}, & \text { otherwise }
\end{array}\right.
$$

be the unit directional vector of the edge $(u, v)$ and define

$$
\begin{aligned}
K(u):= & \mathbf{F}\left(G_{u}\right)-\mathbf{F}(\bar{G}) \\
= & m_{\beta}(u) H_{\beta}\left(\vec{n}_{u}, \vec{e}_{u}\right)\left[\sum_{c h \in C_{v}}\left(\frac{\operatorname{child}(c h)}{m_{\beta}(c h)}+w\left(e_{c h}\right)^{\alpha}|v-c h|\right) H_{\beta}\left(\vec{n}_{u}, \vec{e}_{c h}\right)\right. \\
& \left.+(\text { weight })^{\alpha}|v-u|\right]+P_{G_{u}}(\text { weight }, u),
\end{aligned}
$$

where the number weight is given in (2.8). Here $K(u)$ measures the increment of cost to $\bar{G}$ if we reset the parent of $v$ to $u$. The second identity follows from a direct calculation of the $\mathbf{F}$ costs.

Suppose $K$ achieves its minimum at $u_{0} \in Q_{v}$. Then, we reset the parent of $v$ to be $u_{0}$. Thus, we get a new graph $G_{u_{0}}$. We still denote $G_{u_{0}}$ by $G$.

Step 3. Repeat Step 2 for all $v \in V(G)$. Denote the final modified graph by $\bar{G}$.

Step 4. Repeat the above procedure until the $\operatorname{cost} \mathbf{F}(G)=\mathbf{F}(\bar{G})$. 


\section{The Growth of A tree LEAF}

\subsection{The selection principle}

When a leaf grows, it tends to enlarge its surface area as large as possible. The biological process may be done by separating original cells into new cells in some complicated way. However, its mathematical result is to generate some additional area nearby the boundary in some clever way. As a result, we may assume these additional areas are represented by some new cells that are generated nearby the boundary of the previous stage. The question is: how to select these new cells?

Let

$$
\mathcal{A}_{h}:=\left\{\begin{array}{c}
(\Omega, G): \Omega \subset \Gamma_{h}, G \text { is an optimal transport } \\
\text { system of } \Omega \text { under the } \mathbf{F} \text { cost }
\end{array}\right\} .
$$

For any $(\Omega, G) \in \mathcal{A}_{h}$, how should it generate new cells around its boundary? The choice of the positions of those new cells is not random. For each potential new cell outside the existing leaf $\Omega$, if it is selected as a new cell, it will produce some solar energy. The amount of such kind of revenue is about the same, and proportional to its area. However, the expense corresponding to each potential new cell varies with respect to the position of the cell. Here, the expense is mainly the transport cost of water and nutrients between the cell and the root. Our selection principle says that a new cell is generated only if the expense is less than the revenue it produces. This simple rule determines the selection of new cells during the generation process.

To perform this idea, we first quantify the transporting cost for each cell outside $\Omega$.

For any $(\Omega, G) \in \mathcal{A}_{h}$, a point $q \in \Omega$ is called a boundary point of $\Omega$ if at least one of its eight neighboring cells in $\Gamma_{h}$ is not in $\Omega$. Let

$$
B=\{\text { boundary points of } \Omega\} \cup\{O\} .
$$

For any $x \in \Gamma_{h} \backslash \Omega$, and $b \in B$, we define

$$
C_{\Omega}(x, b):=h^{2 \alpha}|x-b| m_{\beta}(b) H_{\beta}\left(\frac{x-b}{|x-b|}, \vec{e}_{b}\right)+P_{G}\left(h^{2}, b\right),
$$

and set

$$
\begin{aligned}
C_{\Omega}(x) & :=\min _{b \in B} C_{\Omega}(x, b) \\
& =C_{\Omega}(x, b(x)),
\end{aligned}
$$

for some $b(x) \in B$, where $P_{G}$ is the potential function of $G$ defined in $(2.7)$ and $|\cdot|$ is the standard Euclidean distance in $\mathbb{R}^{2}$. The function $C_{\Omega}(x)$ measures the additional transporting cost it needed if one adds a cell located at $x$ and of mass $h^{2}$ to the original existing transport system $G$.

Now, we generate new cells from $(\Omega, G)$ by choosing those cells whose transporting cost is less than a given number. For any given $\epsilon>0$, let

$$
\tilde{\Omega}=\left\{x \in \Gamma_{h} \backslash \Omega: C_{\Omega}(x) \leq \epsilon h^{2}\right\} \cup \Omega
$$

That is, we add those cells to $\Omega$ whose transporting cost $C_{\Omega}(x)$ is less than a given number $\epsilon h^{2}$. Here $C_{\Omega}(x)$ plays the role of "expense" while $\epsilon h^{2}$ plays the role of "revenue". So, $\tilde{\Omega}$ contains those new cells at which revenue exceeds expense.

After selecting these new cells, the leaf tends to develop an efficient transport system for $\tilde{\Omega}$. Let

$$
\begin{gathered}
\tilde{V}=V(G) \cup \tilde{\Omega} \\
\bar{E}=E(G) \cup\{[x, b(x)]: x \in \tilde{\Omega} \backslash \Omega\}
\end{gathered}
$$


and $\bar{w}: \bar{E} \rightarrow(0,+\infty)$ be the weight function determined by $\bar{E}$ and $\tilde{V}$ as in (2.4). Then $\bar{G}=\{\tilde{V}, \bar{E}, \bar{w}\}$ is a transport system of $\tilde{\Omega}$, but not necessarily an optimal one. Using the algorithm stated in the previous section, we may get an optimal transport system $\tilde{G}=\{\tilde{V}, \bar{E}, \bar{w}\}$ of $\tilde{\Omega}$ from $\bar{G}$.

Now, for any given $\epsilon>0$, we get a map $L_{\epsilon, h}: \mathcal{A}_{h} \rightarrow \mathcal{A}_{h}$ by letting

$$
L_{\epsilon, h}(\Omega, G)=(\tilde{\Omega}, \tilde{G})
$$

for any $(\Omega, G) \in \mathcal{A}_{h}$, where $\tilde{\Omega}, \tilde{G}$ are constructed as above.

\subsection{The generation map}

We also note that the optimal transport system $\tilde{G}$ might reduce the transporting costs of cells outside $\tilde{\Omega}$. That is, one might have

$$
C_{\tilde{\Omega}}(x)<C_{\Omega}(x)
$$

for some $x \in \Gamma_{h} \backslash \tilde{\Omega} \subset \Gamma_{h} \backslash \Omega$. Thus, it is possible that $C_{\tilde{\Omega}}(x) \leq \epsilon h^{2}$ even though originally $C_{\Omega}(x)>\epsilon h^{2}$. Therefore, under our selection principle, it is also reasonable to select such cells as new cells. This means that we should consider further $L_{\epsilon, h}^{2}(\Omega, G)=L_{\epsilon, h} \circ L_{\epsilon, h}(\Omega, G), L_{\epsilon, h}^{3}(\Omega, G), \ldots$, and so on. A natural question arises: will $L_{\epsilon, h}^{n}(\Omega, G)$ stop growing and stay in a bounded domain when $n$ is large enough? The answer is yes for $\alpha \in(1 / 2,1)$.

To see this, we first give an estimate of the transport cost function $C_{\Omega}(x)$ as follows.

Proposition 3.1. For any $\Omega \subset \Gamma_{h}$ and any $x \in \Gamma_{h} \backslash \Omega$, we have

$$
C_{\Omega}(x) \geq \frac{\alpha(1+\alpha)}{2} \frac{|x|}{\|\Omega\|^{1-\alpha}} h^{2 \alpha}
$$

where $\|\Omega\|$ denotes the number of elements in $\Omega$, and $|\cdot|$ is the standard Euclidean distance in $\mathbb{R}^{2}$. Moreover, if $|x|>\max \{|y|: y \in \Omega\}$, then

$$
C_{\Omega}(x) \geq C_{\alpha}|x|^{2 \alpha-1} h^{2}
$$

where $C_{\alpha}=\frac{\alpha(1+\alpha)}{2(4 \pi)^{1-\alpha}}$.

Proof. It is easy to check that for any $t \geq 1$, we have the inequality

$$
(1+t)^{\alpha}-t^{\alpha} \geq \frac{\alpha(1+\alpha)}{2 t^{1-\alpha}}
$$

Also, for any $x \in \Gamma_{h} \backslash \Omega$, by the balancing equation (2.2), the maximal weight

$$
\max _{u \in E(G)} w\left(e_{u}\right) \leq\|\Omega\| h^{2} .
$$


Thus,

$$
\begin{aligned}
C_{\Omega}(x) & \geq h^{2 \alpha}|x-b(x)|+\sum_{u \in P_{b(x)}} m_{\beta}(u)\left[\left(w\left(e_{u}\right)+h^{2}\right)^{\alpha}-\left(w\left(e_{u}\right)\right)^{\alpha}\right] \operatorname{length}\left(e_{u}\right) \\
& \geq h^{2 \alpha}|x-b(x)|+\frac{\alpha(1+\alpha)}{2} h^{2 \alpha} \sum_{u \in P_{b(x)}}\left(\frac{h^{2}}{w\left(e_{u}\right)}\right)^{1-\alpha} \operatorname{length}\left(e_{u}\right), \text { by (3.5) } \\
& \geq h^{2 \alpha}|x-b(x)|+\frac{\alpha(1+\alpha)}{2} h^{2 \alpha} \sum_{u \in P_{b(x)}} \frac{1}{\|\Omega\|^{1-\alpha}} \operatorname{length}\left(e_{u}\right) \\
& \geq \frac{\alpha(1+\alpha)}{2} h^{2 \alpha} \frac{1}{\|\Omega\|^{1-\alpha}}\left(|x-b(x)|+\sum_{u \in P_{b(x)}} \operatorname{length}\left(e_{u}\right)\right) \\
& \geq \frac{\alpha(1+\alpha)}{2} h^{2 \alpha} \frac{|x|}{\|\Omega\|^{1-\alpha}} .
\end{aligned}
$$

Moreover, if $|x|>\max \{|y|: y \in \Omega\}$, then

$$
|| \Omega|| h^{2} \leq 4 \pi|x|^{2}
$$

which follows from a comparison on area. Therefore,

$$
\begin{aligned}
C_{\Omega}(x) & \geq \frac{\alpha(1+\alpha)}{2} h^{2 \alpha} \frac{|x|}{\|\Omega\|^{1-\alpha}} \\
& \geq \frac{\alpha(1+\alpha)}{2} h^{2} \frac{|x|}{\left(4 \pi|x|^{2}\right)^{1-\alpha}}=\frac{\alpha(1+\alpha)}{2(4 \pi)^{1-\alpha}} h^{2}|x|^{2 \alpha-1}
\end{aligned}
$$

Let $B_{R_{\epsilon}}(O)$ be the closed ball in $\mathbb{R}^{2}$ centered at the origin $O$ and of radius

$$
R_{\epsilon}=\left(\frac{\epsilon}{C_{\alpha}}\right)^{\frac{1}{2 \alpha-1}}
$$

which is independent of $h$. Let

$$
\mathcal{A}_{\epsilon, h}=\left\{(\Omega, G) \in \mathcal{A}_{h}: \Omega \subset B_{R_{\epsilon}}(O)\right\}
$$

The following proposition says that $L_{\epsilon, h}$ maps $\mathcal{A}_{\epsilon, h}$ to itself when $\alpha \in(1 / 2,1)$.

Proposition 3.2. Suppose $\alpha \in(1 / 2,1)$. For any $(\Omega, G) \in \mathcal{A}_{\epsilon, h}$, if $(\tilde{\Omega}, \tilde{G})=L_{\epsilon, h}(\Omega, G)$, then $(\tilde{\Omega}, \tilde{G}) \in \mathcal{A}_{\epsilon, h}$ and

$$
\mathbf{F}(\tilde{G}) \leq \mathbf{F}(G)+\epsilon h^{2}\|\tilde{\Omega} \backslash \Omega\|
$$

Proof. For any $x \in \Gamma_{h}$ with $|x|>R_{\epsilon}$, by the above proposition, we have

$$
\begin{aligned}
C_{\Omega}(x) & \geq C_{\alpha} h^{2}|x|^{2 \alpha-1} \\
& >C_{\alpha} h^{2}\left|R_{\epsilon}\right|^{2 \alpha-1}=\epsilon h^{2}
\end{aligned}
$$


whenever $\alpha>1 / 2$. Thus, $x \notin \tilde{\Omega}$ for any $x \notin B_{R_{\epsilon}}(O)$. Hence $\tilde{\Omega} \subset B_{R_{\epsilon}}(O) \cap \Gamma_{h}$. Also, using the notations above (3.4),

$$
\begin{array}{rlr}
\mathbf{F}(\tilde{G}) & \leq & \mathbf{F}(\bar{G}) \\
& \leq \mathbf{F}(G)+\sum_{x \in \tilde{\Omega} \backslash \Omega} C_{\Omega}(x) \\
& \leq \mathbf{F}(G)+\epsilon h^{2}\|\tilde{\Omega} \backslash \Omega\|
\end{array}
$$

From now on, we assume $\alpha \in(1 / 2,1)$. For any $\left(\Omega_{0}, G_{0}\right) \in \mathcal{A}_{\epsilon, h}$, we may recursively define

$$
\left(\Omega_{n}, G_{n}\right)=L_{\epsilon, h}\left(\Omega_{n-1}, G_{n-1}\right)
$$

for each $n \geq 1$. Since $\Omega_{0} \subset \Omega_{1} \subset \Omega_{2} \subset \ldots \subset B_{R_{\epsilon}}(O) \cap \Gamma_{h}$ and $B_{R_{\epsilon}}(O) \cap \Gamma_{h}$ is finite, we know that there exists an $N$ such that

$$
\Omega_{N}=\Omega_{N+1}=\ldots
$$

Definition 3.3. Suppose $\alpha \in(1 / 2,1)$. For any $\epsilon>0, h>0$, the generation map

$$
g_{\epsilon, h}: \mathcal{A}_{\epsilon, h} \rightarrow \mathcal{A}_{\epsilon, h}
$$

is defined by setting

$$
g_{\epsilon, h}\left(\Omega_{0}, G_{0}\right)=\left(\Omega_{N}, G_{N}\right)
$$

for any $\left(\Omega_{0}, G_{0}\right) \in \mathcal{A}_{\epsilon, h}$, where $\Omega_{N}, G_{N}$ are given as above.

Thus, if $(\Omega, G)=g_{\epsilon, h}\left(\Omega_{0}, G_{0}\right)$, then

$$
(\Omega, G)=L_{\epsilon, h}(\Omega, G)
$$

is a fixed point of the map $L_{\epsilon, h}: \mathcal{A}_{\epsilon, h} \rightarrow \mathcal{A}_{\epsilon, h}$.

Proposition 3.4. Suppose for any $\left(\Omega_{0}, G_{0}\right) \in \mathcal{A}_{\epsilon, h}$ and $(\Omega, G)=g_{\epsilon, h}\left(\Omega_{0}, G_{0}\right)$. Then the total cost of $G$ is bounded by

$$
\mathbf{F}(G) \leq \mathbf{F}\left(G_{0}\right)+\epsilon h^{2}\left\|\Omega \backslash \Omega_{0}\right\| .
$$

Proof. By recursively using Proposition 3.2, for each $n$,

$$
\begin{aligned}
\mathbf{F}\left(G_{n}\right) & \leq \mathbf{F}\left(G_{n-1}\right)+\epsilon h^{2}\left\|\Omega_{n} \backslash \Omega_{n-1}\right\| \\
& \leq \mathbf{F}\left(G_{0}\right)+\epsilon h^{2}\left\|\Omega_{n} \backslash \Omega_{0}\right\| .
\end{aligned}
$$

Since $\Omega=\Omega_{n}$ when $n$ is large enough, we have

$$
\mathbf{F}(G) \leq \mathbf{F}\left(G_{0}\right)+\epsilon h^{2}\left\|\Omega \backslash \Omega_{0}\right\| .
$$

\subsection{A mathematical model of a tree leaf}

Now, we can discuss the growth of a leaf as follows. The initial stage of any leaf is given by

$$
\Omega_{0}=\{O\} \text { and } G_{0}=\{\{O\}, \emptyset,-\} .
$$

That is, $\Omega_{0}$ consists only the root $O$, and $G_{0}$ does not have any edge. Let $\mathbf{F}$ be a fixed cost functional on the collection of all possible transport systems as before. 
Definition 3.5. For any $\epsilon>0$ and $h>0$, a pair $(\Omega, G) \in \mathcal{A}_{\epsilon, h}$ is called an $(\epsilon, h)$ leaf if there exists a list $\left\{\left(\Omega_{n}, G_{n}\right)\right\}_{n=1}^{k}$ of elements in $\mathcal{A}_{h}$ such that for each $n=1,2, \ldots, k$,

$$
\left(\Omega_{n}, G_{n}\right)=g_{\epsilon_{n}, h}\left(\Omega_{n-1}, G_{n-1}\right)
$$

and

$$
(\Omega, G)=\left(\Omega_{k}, G_{k}\right)
$$

for some positive numbers $\epsilon_{i}$ 's satisfying

$$
0<\epsilon_{1}<\epsilon_{2}<\cdots<\epsilon_{k}=\epsilon
$$

\subsection{Computer visualization}

Based on the algorithms discussed in (3.4) and (3.6), we provide the following computer visualization of different leaves. Also, we representing each $x=(m h, n h) \in \Omega$ by the corresponding 2 dimensional cell $C_{m, n}$, given in (2.1).

Example 1. Let $\alpha=0.6, \beta=0.5$. Then a $(5,1)$ leaf is growing as in Figure 1.

Example 2. Let $\alpha=0.5, \beta=0.8$. Then a $(5,1)$ leaf is growing as in Figure 2.

Example 3. The mathematical leaf on the left side of Figure 3 has a typical maple shape. It has 5 lobes radiating out like fingers from the palm of a hand with sinuses between the lobes. It is a typical example of leaves with palmate venation patterns. We also provide a photo of a nature maple leaf on the right side of Figure 3 to give a comparison. There are several similarities between the nature maple leaf and the leaf generated by our algorithm. The shapes of them are similar. Both of them have 5 bigger lobes and two smaller lobes. Also, relative positions of veins as well as their directions are also similar.

Example 4. The mathematical leaf on the left side of Figure 4 is also generated by the above algorithm. It has a typical cordate shape, which is enjoyed by many leaves in nature such as some mulberry leaves. As for its venation pattern, it is a typical example of leaves with pinnate venation patterns. It has one large central vein (midrib) presented with smaller lateral veins that diverge in pairs manner, each on the opposite side of the midrib. These facts agree with the leaf structures we observed in nature. For comparison purpose, we also provide a photo of a nature mulberry leaf on the right side of Figure 4. We actually measured both leaves in Figure 4 and obtain the following data.

\begin{tabular}{|l|c|c|}
\hline & The nature maple leaf & The generated leaf \\
\hline Area $\left(\mathrm{cm}^{2}\right)$ & 10.75 & 11 \\
\hline Length $(\mathrm{cm})$ & 4.5 & 4.5 \\
\hline Width $(\mathrm{cm})$ & 3.5 & 3.2 \\
\hline Length of the midrib $(\mathrm{cm})$ & 4.3 & 4.2 \\
\hline
\end{tabular}

Here we rescaled the photo so that both leaves in Figure 4 have the same length. Note that their area, width and even the length of the midribs are all very close. We also noticed that both leaves have 5 pairs of veins of second order. The length of those veins from the bottom to the top are listed (in centimeters) as follows:

\begin{tabular}{|l|l|l|l|l|l|l|}
\hline & & No. 1 & No. 2 & No. 3 & No. 4 & No. 5 \\
\hline \multirow{2}{*}{$\begin{array}{l}\text { The } \\
\text { nature } \\
\text { leaf }\end{array}$} & The left side veins & 2.84 & 2.45 & 1.89 & 1.53 & 1.14 \\
\cline { 2 - 7 } & The right side veins & 2.68 & 2.52 & 1.93 & 1.50 & 0.95 \\
\cline { 2 - 7 } & Average of both sides & 2.76 & 2.48 & 1.91 & 1.52 & 1.05 \\
\hline Generated leaf & Both sides & 2.2 & 2.0 & 1.7 & 1.2 & 0.9 \\
\hline
\end{tabular}



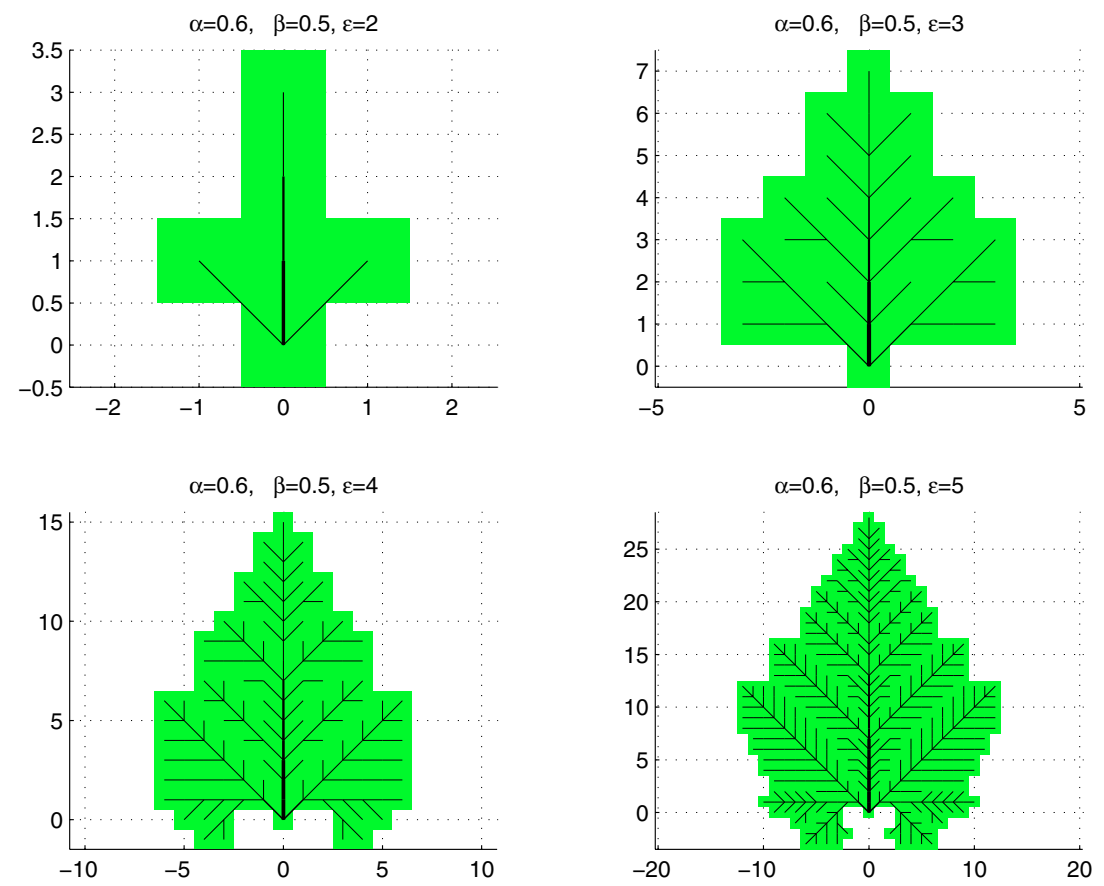

Figure $1 . \alpha=0.6, \beta=0.5$.
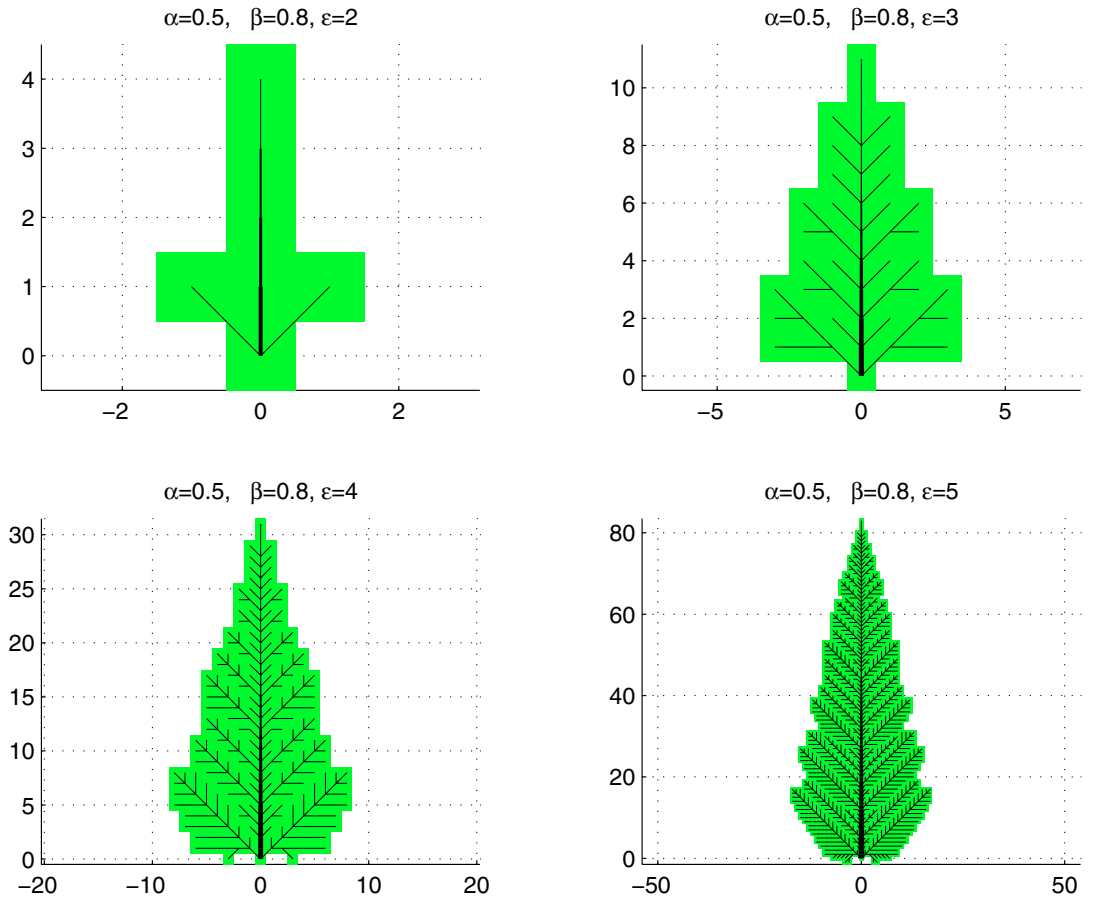

FiguRE 2. $\alpha=0.5, \beta=0.8$. 

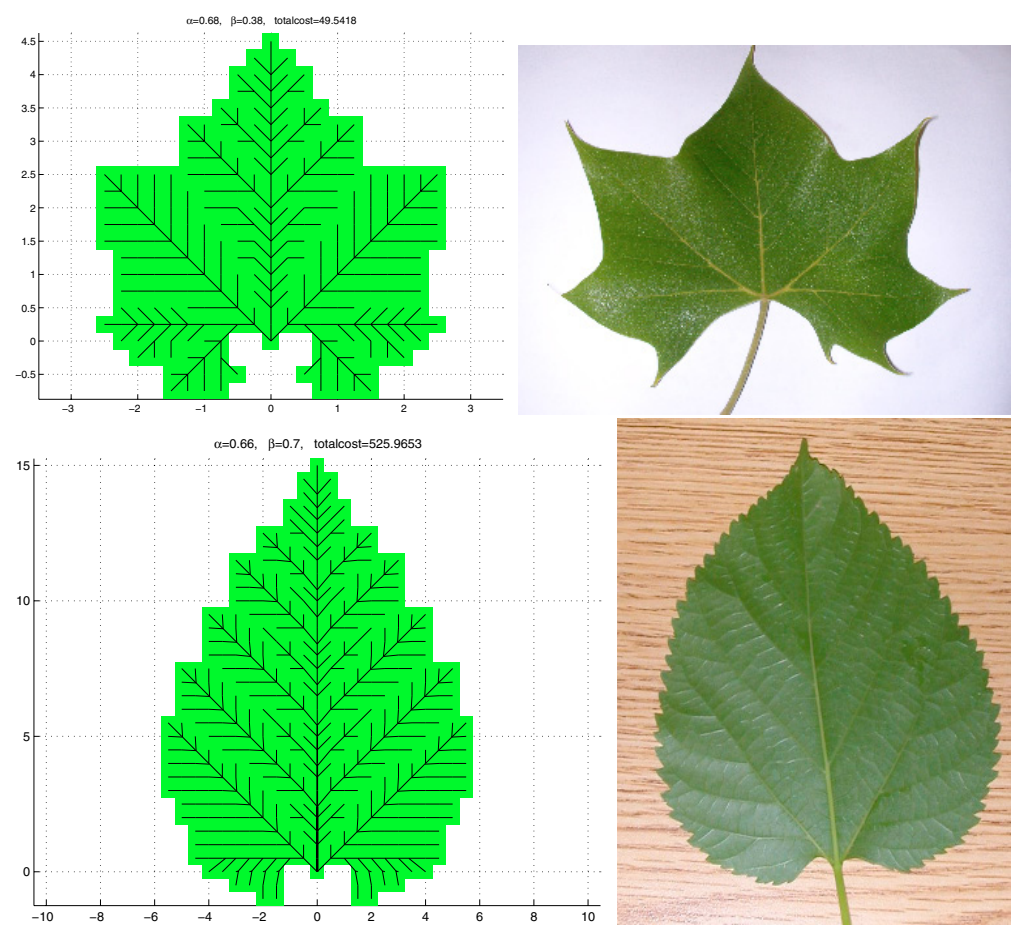

Figure 3. A maple leaf.
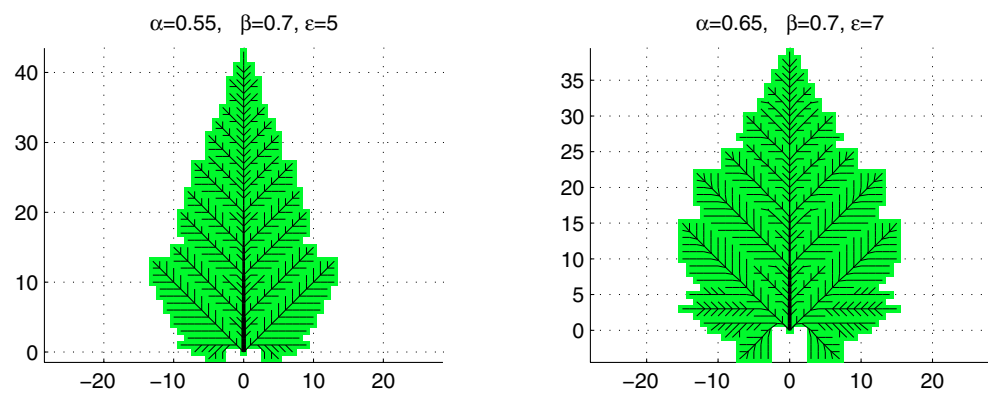

$\alpha=0.75, \quad \beta=0.7, \varepsilon=9$
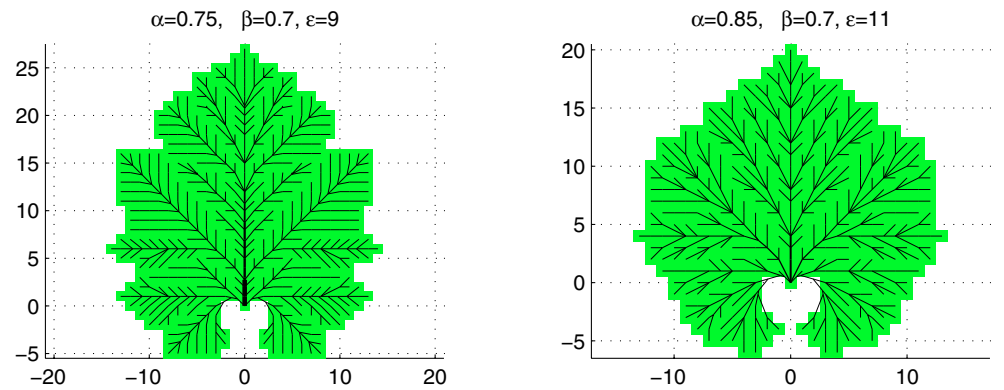

FigURE 4. Figures of various parameters. 


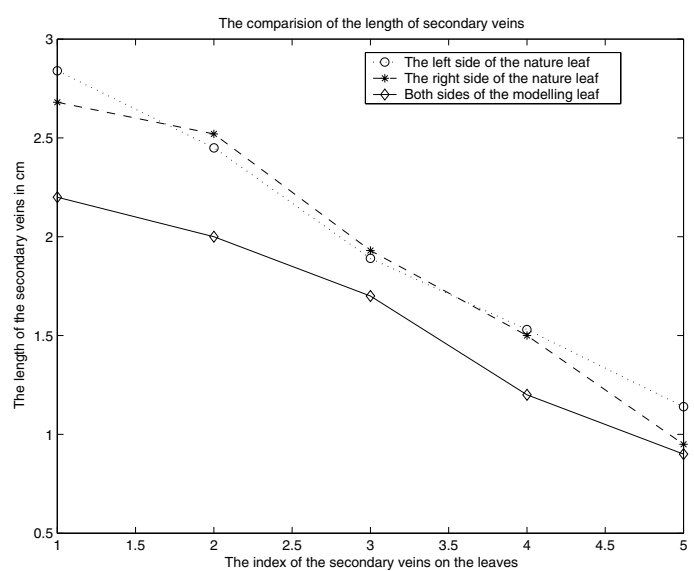

FiguRE 5. A comparison on the length of veins of second order.

A plot of these data is given in Figure 6 .

From these data, we can compute the ratio of length of adjacent veins. For the photoed leaf, we use the average data of both sides.

\begin{tabular}{|c|c|c|c|c|c|}
\hline & No. 1/No. 2 & No. 2/No. 3 & No. 3/No. 4 & No. 4/No. 5 & Average \\
\hline Nature leaf & 1.11 & 1.29 & 1.26 & 1.46 & 1.28 \\
\hline Generated leaf & 1.10 & 1.18 & 1.42 & 1.33 & 1.26 \\
\hline
\end{tabular}

Note that the average ratio of them are very close to each other.

Example 5. The final shape of a leaf depends on the cost functional $\mathbf{F}$ one chooses for transport systems. Changing the values of the parameters $\alpha$ and $\beta$ will give us leaves of different shapes. The Figure 5 illustrates this phenomenon by changing the values of $\alpha$ while fixing the values of $\beta$.

Remark 3.6. Recall that $\epsilon h^{2}$ in (3.3) plays the role of "revenue" generated at each cell. Here, for simplicity, we assume $\epsilon$ to be constant on all cells at a given time. The above process gives us a "symmetric leaf". If one would like to take the environment factor into consideration, he may also allow $\epsilon$ to have a noising term. For instance, cells on the leaf may be exposed under the sun unevenly. Some of them maybe shadowed. In such cases, $\epsilon$ should not be treated as a constant. The same process as above will provide a usually "non-symmetric" leaf. Moreover, if one let $\epsilon$ to be a periodic function of time, we may get leaves in different seasons. The above process may be used to predict which cells will die first, and then predict a dynamic coloring process of the leaf in seasons.

Remark 3.7. Under the same conditions (i.e. same $\beta$, same $\epsilon$ ), leaves will become rounder as the parameter $\alpha$ increase. For $\alpha$ nearby 0, one would get a "grass-like" leaf. As $\alpha$ approaches 1, one would get a more round leaf.

The following two simple lemmas will be useful in the following sections.

Lemma 3.8. For each $(\epsilon, h)$ leaf $(\Omega, G)$, we have

$$
\mathbf{F}(G) \leq \epsilon h^{2}\|\Omega\| .
$$

Proof. It follows from repeatedly using Proposition 3.4 and the fact $\mathbf{F}\left(G_{0}\right)=0$. 
Lemma 3.9. For each $(\epsilon, h)$ leaf $(\Omega, G)$, there is no decomposition of $\Omega$ such that $\Omega=X \cup Y$ for some subsets $X, Y \subset \Gamma_{h}$ with

$$
\operatorname{dist}(X, Y)>\sqrt{2} h,
$$

where $\operatorname{dist}(X, Y)=\min \{|x-y|: x \in X$ and $y \in Y\}$.

Proof. This follows from the construction of the $(\epsilon, h)$ leaf.

\subsection{A limit process}

Let $h>0$ be a fixed number. For any nondecreasing sequence $\vec{\epsilon}$ :

$$
0<\epsilon_{1} \leq \epsilon_{2} \leq \cdots \leq \epsilon_{k} \leq \epsilon_{k+1} \leq \cdots
$$

of positive numbers, we can get a sequence of $\left(\epsilon_{k}, h\right)$ leaves by letting

$$
\left(\Omega_{k}, G_{k}\right)=g_{\epsilon_{k}, h}\left(\Omega_{k-1}, G_{k-1}\right)
$$

for each $k$. We are interested in the asymptotic behavior of the shapes of $\left(\Omega_{k}, G_{k}\right)$ as $k$ approaches $\infty$.

By Propositions 3.2 and 3.4, we have

$$
\begin{gathered}
\Omega_{0} \subset \Omega_{1} \subset \ldots \subset \Omega_{k} \subset \Omega_{k+1} \subset \ldots \\
\Omega_{k} \subset B_{R_{\epsilon_{k}}}(0)
\end{gathered}
$$

and

$$
\mathbf{F}\left(G_{k}\right) \leq \epsilon_{k} h^{2}\left\|\Omega_{k}\right\| .
$$

If the sequence $\vec{\epsilon}$ in (3.8) is bounded above by a finite number $\epsilon$, then for any $k, \Omega_{k}$ is contained in the finite set $B_{R_{\epsilon}}(0) \cap \Gamma_{h}$. Thus, the nested sets $\Omega_{k}$ 's are ultimately equal to each other as sets. That is,

$$
\Omega_{k}=\Omega_{k+1}=\ldots
$$

when $k$ is large enough. In this case, $\left(\Omega_{k}, G_{k}\right)$ is fixed when $k$ is large enough and the leaf will stop growing. Indeed, in realty, $\vec{\epsilon}$ should be bounded. This explains why tree leaves will stop growing.

In the case $\vec{\epsilon}$ is unbounded, the situation is no longer so simple, for the radius $R_{\epsilon_{k}}$ will approach $\infty$ as $k$ approaches $\infty$. Later, we will see that this case is also corresponding to the problem of fixing $\epsilon$ but let the step size $h$ approaches 0 . To understand this situation, we first view each $\Omega_{k}$ (or $G_{k}$ ) as a Radon measure (or vector measure) on $\mathbb{R}^{2}$, rescale it into a fixed ball, and then take the limit as Radon measure in that ball.

Let $\mathcal{M}$ be the space of Radon measures on $\mathbb{R}^{2}$, and let $\mathcal{M}^{2}$ be the space of vector measures $\mu=\left(\mu_{1}, \mu_{2}\right)$ on $\mathbb{R}^{2}$. We consider the map

$$
\begin{gathered}
\phi_{h}: \mathcal{A}_{h} \rightarrow \mathcal{M} \times \mathcal{M}^{2} \\
(\Omega, G) \rightarrow(\mu, \Theta)
\end{gathered}
$$

for each $(\Omega, G) \in \mathcal{A}_{h}$, where

$$
\mu:=\sum_{x \in \Omega} h^{2} \delta_{x}
$$

is an atomic Radon measure on $\mathbb{R}^{2}$, and

$$
\Theta=\sum_{e \in E(G)} w(e) \mathcal{H}^{1}\lfloor e \vec{e}
$$


is a vector measure on $\mathbb{R}^{2}$. Here $\mathcal{H}^{1} L_{e}$ is the one dimensional Hausdorff measure on $e$ for each edge $e \in E(G)$ with unit directional vector $\vec{e}$. The system of balancing equations (2.2) can be simplified to be a single divergence condition on the vector measure $\Theta$

$$
\operatorname{div}(\Theta)=\mathbf{M}(\mu) \delta_{O}-\mu
$$

in the sense of distribution, where $\mathbf{M}(\mu)=\mu\left(\mathbb{R}^{2}\right)$ denotes the total mass of $\mu$.

Lemma 3.10. Suppose $(\Omega, G)$ is an $(\epsilon, h)$ leaf and $(\mu, \Theta)=\phi_{h}(\Omega, G)$. Then the total mass of the Radon measure is bounded above by

$$
\mathbf{M}(\mu) \leq \pi\left(R_{\epsilon}+h\right)^{2}
$$

and the total variation of the vector measure $\Theta$ is bounded by

$$
\mathbf{M}(\Theta) \leq \epsilon \pi^{2-\alpha}\left(R_{\epsilon}+h\right)^{4-2 \alpha} .
$$

Proof. Since $\Omega \subset B_{R_{\epsilon}}(O)$, the mass of $\mu$ is given by

$$
\begin{aligned}
\mathbf{M}(\mu) & =\|\Omega\| h^{2} \\
& =\operatorname{area}\left(\bigcup_{x \in \Omega}\left\{x+\left[-\frac{h}{2}, \frac{h}{2}\right] \times\left[-\frac{h}{2}, \frac{h}{2}\right]\right\}\right) \\
& \leq \operatorname{area}\left(B_{R_{\epsilon}+h}(0)\right)=\pi\left(R_{\epsilon}+h\right)^{2} .
\end{aligned}
$$

Also, since $w(e) \leq\|\Omega\| h^{2}$ for each $e \in E(G)$, the total variation of $\Theta$ is given by

$$
\begin{aligned}
\mathbf{M}(\Theta) & =\sum_{e \in E(G)} w(e) \text { length }(e) \\
& \leq\left(\|\Omega\| h^{2}\right)^{1-\alpha} \sum_{e \in E(G)} m_{\beta}\left(e^{+}\right)(w(e))^{\alpha} \text { length }(e) \\
& =\left(\|\Omega\| h^{2}\right)^{1-\alpha} \mathbf{F}(G) \leq \epsilon\left(\|\Omega\| h^{2}\right)^{2-\alpha}, \text { by Lemma } 3.8 \\
& \leq \epsilon \pi^{2-\alpha}\left(R_{\epsilon}+h\right)^{2(2-\alpha)} .
\end{aligned}
$$

On the other hand, we also consider the rescaling of $(\epsilon, h)$ leaf as follows. For each $\lambda>0$, let $\varphi_{\lambda}(x)=\lambda x$ for each $x \in \mathbb{R}^{2}$ be the scaling map. Then $\varphi_{\lambda}$ induces a push forward map $\varphi_{\lambda \#}: \mathcal{A}_{h} \rightarrow \mathcal{A}_{\lambda h}$ by sending each $(\Omega, G)$ to $\varphi_{\lambda \#}(\Omega, G)=\left(\Omega^{\lambda}, G^{\lambda}\right)$, where

$$
\Omega^{\lambda}=\{\lambda x: x \in \Omega\} \subset \Gamma_{\lambda h}
$$

and $G^{\lambda}=\left\{V^{\lambda}, E^{\lambda}, w^{\lambda}\right\}$ with

$$
\begin{aligned}
V^{\lambda} & =\{\lambda u: u \in V(G)\} \\
E^{\lambda} & =\{(\lambda u, \lambda v):(u, v) \in E(G)\} \\
\text { and } w^{\lambda}(\lambda u, \lambda v) & =\lambda^{2} w(u, v) \text { for each }(u, v) \in E(G) .
\end{aligned}
$$

This push forward map is consistent with the push forward map $\left(\varphi_{\lambda}\right)_{\#}$ of Radon measures in the sense that

$$
\phi_{\lambda h} \circ\left(\varphi_{\lambda}\right)_{\#}(\Omega, G)=\left(\varphi_{\lambda}\right)_{\#} \circ \phi_{h}(\Omega, G) .
$$

Lemma 3.11. For any $\epsilon>0$ and $\lambda>0$, we have

$$
\varphi_{\lambda \#} \circ g_{\epsilon, h}=g_{\lambda^{2 \alpha-1} \epsilon, \lambda h} \circ \varphi_{\lambda \#} .
$$


Proof. For any $(\Omega, G) \in \mathcal{A}_{h}$ and any $x \in \Gamma_{h} \backslash \Omega$, we have

$$
C_{\varphi_{\lambda \#}(\Omega, G)}(\lambda x)=\lambda^{1+2 \alpha} C_{\Omega}(x) .
$$

Thus, for any $\epsilon>0$,

$$
C_{\Omega}(x) \leq \epsilon h^{2} \Leftrightarrow C_{\varphi_{\lambda \#}(\Omega, G)}(\lambda x) \leq \lambda^{1+2 \alpha} \epsilon h^{2}=\left(\lambda^{2 \alpha-1} \epsilon\right)(\lambda h)^{2},
$$

which implies that

$$
\varphi_{\lambda \#}\left(L_{\epsilon, h}(\Omega, G)\right)=L_{\lambda^{2 \alpha-1} \epsilon, \lambda h}\left(\varphi_{\lambda \#}(\Omega, G)\right)
$$

and hence

$$
\varphi_{\lambda \#} \circ g_{\epsilon, h}(\Omega, G)=g_{\lambda^{2 \alpha-1} \epsilon, \lambda h} \circ \varphi_{\lambda \#}(\Omega, G) .
$$

Using this lemma and Definition 3.5 of $(\epsilon, h)$ leaves, we have

Corollary 3.12. For any $\lambda>0, \varphi_{\lambda \#}$ maps any $(\epsilon, h)$ leaf $(\Omega, G)$ to another $\left(\lambda^{2 \alpha-1} \epsilon, \lambda h\right)$ leaf $\left(\varphi_{\lambda}\right)_{\#}(\Omega, G)$.

Now, let $0<\epsilon_{1}<\epsilon_{2}<\cdots<\epsilon_{k}<\epsilon_{k+1}<\cdots$ be any unbounded increasing sequence of real numbers, and let

$$
\left(\Omega_{k}, G_{k}\right)=g_{\epsilon_{k}, h}\left(\Omega_{k-1}, G_{k-1}\right)
$$

for each $k=1,2, \ldots$ as before. To understand the asymptotic behavior of the shapes of the $\left(\epsilon_{k}, h\right)$ leaves $\left(\Omega_{k}, G_{k}\right)$ as $k$ approaches $\infty$, we let

$$
\left(\tilde{\Omega}_{k}, \tilde{G}_{k}\right)=\varphi_{\lambda_{k} \#}\left(\Omega_{k}, G_{k}\right)
$$

for each $k$, where $\lambda_{k}=\epsilon_{k}^{\frac{1}{1-2 \alpha}} \rightarrow 0$ as $k$ approaches $\infty$.

Lemma 3.13. For each $k,\left(\tilde{\Omega}_{k}, \tilde{G}_{k}\right)$ satisfies the following properties:

(1) $\left(\tilde{\Omega}_{k}, \tilde{G}_{k}\right)$ is a $\left(1, \lambda_{k} h\right)$ leaf.

(2) $\tilde{\Omega}_{k} \subset B_{R_{1}}(0)$ is uniformly bounded.

(3) $\left(\tilde{\Omega}_{k}, \tilde{G}_{k}\right)$ is a rescale of $\left(\Omega_{k}, G_{k}\right)$, thus their shapes are similar.

(4) $\left(\tilde{\Omega}_{k+1}, \tilde{G}_{k+1}\right)=g_{1, \lambda_{k+1} h}\left(\varphi_{\frac{\lambda_{k+1}}{\lambda_{k}}}\right)_{\#}\left(\tilde{\Omega}_{k}, \tilde{G}_{k}\right)$.

Proof. (1) follows from the previous corollary. (2) follows from (1). (3) follows from Definition 3.11 and (4) follows from Lemma 3.11 and

$$
\begin{aligned}
& \left(\tilde{\Omega}_{k+1}, \tilde{G}_{k+1}\right)=\varphi_{\lambda_{k+1} \#}\left(\Omega_{k+1}, G_{k+1}\right) \\
& =\varphi_{\lambda_{k+1} \#} g_{\epsilon_{k+1}, h}\left(\Omega_{k}, G_{k}\right) \\
& =\varphi_{\lambda_{k+1} \#} g_{\epsilon_{k+1}, h} \varphi_{\lambda_{k}^{-1} \#}\left(\tilde{\Omega}_{k}, \tilde{G}_{k}\right) \\
& =g_{\lambda_{k+1}^{2 \alpha-1} \epsilon_{k+1}, \lambda_{k+1} h} \varphi_{\lambda_{k+1} \#} \varphi_{\lambda_{k}^{-1} \#}\left(\tilde{\Omega}_{k}, \tilde{G}_{k}\right) \\
& =g_{1, \lambda_{k+1} h}\left(\varphi_{\frac{\lambda_{k+1}}{\lambda_{k}}}\right)_{\#}\left(\tilde{\Omega}_{k}, \tilde{G}_{k}\right) \text {. }
\end{aligned}
$$


Thus, to understand the final shapes of $\left(\epsilon_{k}, h\right)$ leaves $\left(\Omega_{k}, G_{k}\right)$ as $k \rightarrow \infty$, it is sufficient to understand the final shapes of $\left(1, \lambda_{k} h\right)$ leaves $\left(\tilde{\Omega}_{k}, \tilde{G}_{k}\right)$ as $k \rightarrow \infty$. To take the limit of $\left(1, \lambda_{k} h\right)$ leaves $\left(\tilde{\Omega}_{k}, \tilde{G}_{k}\right)$, we consider the associated Radon measures

$$
\left(\mu_{k}, \Theta_{k}\right)=\phi_{\lambda_{k} h}\left(\tilde{\Omega}_{k}, \tilde{G}_{k}\right)=\left(\varphi_{\lambda_{k}}\right)_{\#}\left(\phi_{h}(\Omega, G)\right)
$$

for each $k$. By Lemma 3.10, for each $k$,

$$
\mathbf{M}\left(\mu_{k}\right) \leq \pi\left(R_{1}+\lambda_{k} h\right)^{2} \rightarrow \pi R_{1}^{2}
$$

as $k \rightarrow+\infty$ and

$$
\mathbf{M}\left(\Theta_{k}\right) \leq \pi\left(R_{1}+\lambda_{k} h\right)^{2(2-\alpha)} \rightarrow \pi R_{1}^{2(2-\alpha)}
$$

as $k \rightarrow+\infty$. Therefore,

$$
\mathbf{M}\left(\mu_{k}\right)+\mathbf{M}\left(\Theta_{k}\right)
$$

is uniformly bounded above.

Proposition 3.14. Let $\left(\Omega_{n}, G_{n}\right)$ be defined as in (3.10), and let $\left(\mu_{n}, \Theta_{n}\right)$ be the corresponding measures and vector measures as in (3.12). Then $\mu_{n}$ is weakly subconvergent to a Radon measure $\mu$ on $B_{R_{1}}(O)$ and $\Theta_{n}$ is weakly subconvergent to a vector measure $\Theta$ on $B_{R_{1}}(O)$ such that

$$
\operatorname{div}(\Theta)=\mathbf{M}(\mu) \delta_{O}-\mu
$$

in the sense of distribution. Moreover, the support of $\mu$ is a connected compact set contained in $B_{R_{1}}(0)$.

Proof. As discussed above, the total mass of $\mu_{n}$ and the total variation of $\Theta_{n}$ are uniformly bounded. Thus, by the compactness of Radon measures and vector measures, $\Theta_{n}$ and $\mu_{n}$ are weakly subconvergent to a vector measure $\Theta$ and a Radon measure $\mu$ on $B_{R_{1}}(0)$. Moreover, by (3.9), we have

$$
\operatorname{div}(\Theta)=\mathbf{M}(\mu) \delta_{O}-\mu
$$

in the sense of distribution. Also, by definition, the support $\operatorname{spt}(\mu)$ of $\mu$ is a compact subset of $B_{R_{\epsilon}}(0)$.

Assume $\operatorname{spt}(\mu)$ is not connected, then one can decompose $\operatorname{spt}(\mu)$ as the union of two closed subsets $X, Y$ :

$$
\operatorname{spt}(\mu)=X \cup Y
$$

such that

$$
\operatorname{dist}(X, Y) \geq t
$$

for some $t>0$, where $\operatorname{dist}(X, Y)=\min \{|x-y|: x \in X$ and $y \in Y\}$. For any $n$ with $\lambda_{n}<\frac{t}{2 h}$, since $\Omega_{n} \subset$ $\operatorname{spt}(\mu)$,

$$
\Omega_{n}=\left(\Omega_{n} \cap X\right) \cup\left(\Omega_{n} \cap Y\right)
$$

and

$$
\operatorname{dist}\left(\Omega_{n} \cap X, \Omega_{n} \cap Y\right) \geq t>\sqrt{2} \lambda_{n} h,
$$

which is impossible by Lemma 3.9. Therefore, $\operatorname{spt}(\mu)$ is connected. 


\section{REFERENCES}

[1] M. Bernot, V. Caselles and J.-M. Morel, Are there infinite irregation tree? J. Math. Fluid Mech. 8 (2006) 311-332.

[2] A. Brancolini, G. Buttazzo and F. Santambrogio, Path functions over Wasserstein spaces. http://www.calcvar.sns.it/ papers/brabutsan04/path.pdf

[3] T. De Pauw and R. Hardt, Size minimization and approximating problems. Calc. Var. Partial Differ. Equ. 17 (2003) $405-442$.

[4] von C. Ettingshausen, Die Blatt-Skelete der Dikotyledonen. Wien: Staatsdruckerei, Wien (1861).

[5] E.N. Gilbert, Minimum cost communication networks. Bell System Tech. J. 46 (1967) 2209-2227.

[6] J.M. Harris, J.L. Hist and M.J. Mossinghoff, Combinatorics and graph theory. Springer-verlag (2000).

[7] L.J. Hickey, A revised classification of the architecture of dicotyledonous leaves, in Anatomy of the dicotyledons, 2nd edn., Vol. I, Systematic anatomy of the leaves and stem., C.R. Metcalfe, L. Chalk, Eds., Oxford, Clarendon Press (1979) $25-39$.

[8] F. Maddalena, J.-M. Morel and S. Solimini, A variational model of irrigation patterns. Interfaces Free Bound. 5 (2003) $391-415$.

[9] R. Melville, Leaf venation patterns and the origin of angiosperms. Nature 224 (1969) 121-125.

[10] R. Melville, The terminology of leaves architecture. Taxon 25 (1976) 549-562.

[11] T. Nelson and N. Dengler, Leaf vascular pattern formation. Plant Cell 9 (1997) 1121-1135.

[12] Q. Xia, Optimal paths related to transport problems. Comm. Cont. Math. 5 (2003) 251-279.

[13] Q. Xia, Interior regularity of optimal transport paths. Calc. Var. Partial Differ. Equ. 20 (2004) 283-299.

[14] Q. Xia, Boundary regularity of optimal transport paths. http://math.ucdavis.edu/ qlxia/Research/boundary.pdf. 